\title{
Factors Associated With Early Recovery of Stress Urinary Incontinence Following Holmium Laser Enucleation of the Prostate in Patients With Benign Prostatic Enlargement
}

\author{
Kang Jun Cho, Jun Sung Koh, Jin Bong Choi, Joon Chul Kim \\ Department of Urology, Bucheon St. Mary's Hospital, College of Medicine, The Catholic University of Korea, Bucheon, Korea
}

Purpose: To investigate factors associated with early recovery of stress urinary incontinence (SUI) following holmium laser enucleation of the prostate (HoLEP) in patients with benign prostatic enlargement (BPE).

Methods: The medical records of 393 patients who underwent HoLEP for BPE were retrospectively reviewed. Patients with SUI following HoLEP were selected and divided into 2 groups: those who experienced early recovery of SUI and those who experienced persistent SUI. Recovery of SUI within 1 month after HoLEP was defined as early, and SUI that remained present after 1 month was defined as persistent. Preoperative clinical and urodynamic factors, as well as perioperative factors, were compared between groups.

Results: SUI following HoLEP was detected in 86 patients. Thirty-three patients exhibited recovery of SUI within 1 month, and SUI remained present in 53 patients after 1 month. Multivariate analysis showed that the transition zone prostate volume (odds ratio $[\mathrm{OR}], 5.354 ; 95 \%$ confidence interval $[\mathrm{CI}], 1.911-14.999 ; \mathrm{P}=0.001)$ and the enucleation ratio (OR, 8.253; 95\% $\mathrm{CI}$, $1.786-38.126 ; \mathrm{P}=0.007$ ) were significantly associated with early recovery of SUI.

Conclusions: Early recovery of SUI within 1 month following HoLEP was associated with transition zone prostate volume and the enucleation ratio.

Keywords: Benign prostatic hyperplasia; Holmium lasers; Stress urinary incontinence

- Research Ethics: This study was approved by the Bucheon St. Mary's Hospital ethics committee of the Catholic University of Korea (HIRB-

20171030-002). This study was exempt from consent from the ethics committee because it is a study using previously recorded data.

- Conflict of Interest: No potential conflict of interest relevant to this article was reported.

\section{INTRODUCTION}

Although the most commonly reported etiology of stress urinary incontinence (SUI) in males is postradical prostatectomy, surgery for benign prostatic enlargement (BPE) accounts for approximately $10 \%$ of cases of male SUI [1]. There are several surgical procedures for BPE. Holmium laser enucleation of the prostate (HoLEP) has shown good treatment outcomes and safety compared to alternative surgical procedures for the management of BPE without any limitation on prostate size. However, operators should be more concerned about SUI when performing HoLEP. Although the incidence of SUI has been reported as 3\%-9\% after open prostatectomy [2,3] and approximately $2 \%$ after transurethral resection of the prostate (TURP)

Corresponding author: Joon Chul Kim (D) https://orcid.org/0000-0002-4019-620X Department of Urology, Bucheon St. Mary's Hospital, College of Medicine, The Catholic University of Korea, 327 Sosa-ro, Wonmi-gu, Bucheon 14647, Korea E-mail: kjc@catholic.ac.kr / Tel: +82-32-340-7071 / Fax: +82-32-340-2124 Submitted: April 10, 2018 / Accepted after revision: August 25, 2018

This is an Open Access article distributed under the terms of the Creative Commons Attribution Non-Commercial License (http://creativecommons.org/licenses/by-nc/4.0/) which permits unrestricted non-commercial use, distribution, and reproduction in any medium, provided the original work is properly cited. 
[4], few studies have investigated the incidence of SUI after open prostatectomy or TURP. However, the incidence of SUI following HoLEP has been reported in several studies as 4.9\%$12.5 \%$ [5-7]. The factors possibly associated with SUI following HoLEP include the surgeon's experience, the presence of diabetes mellitus, large prostate volume, greater reduction in postoperative prostate-specific antigen levels, longer operation time, longer enucleation time, and increased blood loss [5,8-10]. SUI following HoLEP improves spontaneously within 3 months in $70 \%-90 \%$ of patients, and studies of transient SUI usually focus on the 3-month postoperative period [5,8]. Although physicians can counsel patients that SUI will improve within 3 months, this information usually causes significant stress and anxiety for the patient and decreases the patient's quality of life throughout the duration of SUI. Therefore, it would be helpful to be able to predict whether SUI will improve sooner rather than later. We investigated factors associated with early recovery of SUI following HoLEP in patients with BPE.

\section{MATERIALS AND METHODS}

The present study retrospectively analyzed the medical records of 393 patients who underwent HoLEP for BPE. The included patients had at least 3 months of postoperative follow-up data, and the following exclusion criteria were applied: a diagnosis of prostate cancer before or after HoLEP based on prostate biopsy, a history of prior prostatic and/or urethral surgery, a suspected neurogenic cause of voiding symptoms, and the presence of preoperative SUI. Two experienced surgeons (JCK or KJC) who had performed more than 20 HoLEP procedures performed all operations at our center. The procedures were conducted using an 80-W holmium laser (2 J/40 Hz setting) following Gilling's technique with minimal variation [11]. Transrectal needle biopsies of the prostate were performed to exclude prostate cancer when clinically indicated.

Patients with SUI following HoLEP who were evaluated by a medical interview and the Marshall test at 2 weeks postoperatively were selected. SUI status was evaluated at 1 month and 3 months postoperatively. Patients were divided into 2 groups: those who experienced early recovery of SUI and those who experienced persistent SUI. Recovery of SUI within 1 month after HoLEP was defined as early, and SUI that remained present after 1 month was defined as persistent. Continence was defined as complete dryness, and the definition of SUI included both stress and mixed urinary incontinence. Preoperative clini- cal and urodynamic factors, as well as perioperative factors, were compared between the groups. The following preoperative clinical factors were investigated: age, history of acute urinary retention, urgency incontinence, serum prostate-specific antigen levels, total prostate volume, transition zone (TZ) prostate volume, and International Prostate Symptom Score (IPSS). Prostate volume was measured via transrectal ultrasonography. Urodynamic parameters were also compared between the 2 groups, and included maximum flow rate (Qmax), postvoid residual urine volume (PVR), maximum bladder capacity, maximum urethral closure pressure, detrusor pressure at Qmax, the bladder outlet obstruction (BOO) index, $\mathrm{BOO}$ grade, and bladder contractility index. The BOO grade was measured using the Schafer obstruction grade. The perioperative results included enucleation time, morcellation time, laser energy used, enucleation weight, enucleation efficiency, morcellation efficiency, and enucleation ratio (enucleation weight/TZ volume). We used prostate ellipse volume calculation to measure the prostate volume on transrectal ultrasound.

Continuous variables were reported as mean and standard deviation, and categorical variables were expressed as frequencies and percentages. The clinical and urodynamic characteristics were evaluated to identify statistically significant differences between the early SUI recovery group and the persistent SUI group using the Student $\mathrm{t}$-test for continuous variables and the chi-square and Fisher exact tests for categorical variables. Logistic regression analysis was used to identify factors influencing the early recovery of SUI. Variables with P-values less than 0.05 in the univariate analysis were included in the multivariate logistic regression model. A 5\% significance level was used for all statistical tests. All statistical analyses were performed using IBM SPSS Statistics ver. 20.0 (IBM Co., Armonk, NY, USA).

\section{RESULTS}

Of the 393 patients who underwent HoLEP, SUI was observed in 86 patients (21.9\%). Thirty-three patients showed recovery of SUI within 1 month, which accounted for $38.4 \%$ (33 of 86) of all patients with SUI and 8.4\% (33 of 393) of all patients who underwent HoLEP. SUI remained present in 53 patients ( 53 of $86,61.6 \%$ ) after 1 month. A total of 18 patients (18 of 86, 20.9\%) had SUI after 3 months, corresponding to $4.6 \%$ (18 of 393) of all patients who underwent HoLEP.

The preoperative clinical characteristics and urodynamic parameters are shown in Table 1. There were more patients with a 
Table 1. Baseline characteristics of patients with postoperative stress urinary incontinence

\begin{tabular}{|c|c|c|c|c|}
\hline Variable & Total $(\mathrm{n}=86)$ & Early SUI recovery $(\mathrm{n}=33)$ & Persistent SUI $(\mathrm{n}=53)$ & P-value \\
\hline Age (yr) & $68.5 \pm 7.2$ & $68.8 \pm 7.2$ & $68.2 \pm 7.2$ & 0.685 \\
\hline A history of AUR & $17(19.8)$ & $8(24.2)$ & $9(17.0)$ & 0.411 \\
\hline Urgency incontinence & $17(19.8)$ & $6(18.2)$ & $11(20.8)$ & 0.771 \\
\hline PSA (ng/mL) & $7.7 \pm 14.4$ & $9.3 \pm 17.5$ & $6.7 \pm 12.3$ & 0.437 \\
\hline Total prostate volume $(\mathrm{mL})$ & $55.1 \pm 22.4$ & $59.9 \pm 23.1$ & $52.1 \pm 21.7$ & 0.114 \\
\hline $\begin{array}{l}\text { Transitional zone volume }(\mathrm{mL}) \\
\quad<30 \\
\geq 30\end{array}$ & $\begin{array}{r}29.1 \pm 16.9 \\
54(62.8) \\
32(37.2)\end{array}$ & $\begin{array}{c}35.0 \pm 19.9 \\
14(42.4) \\
19(57.6)\end{array}$ & $\begin{array}{l}25.4 \pm 13.7 \\
40(75.5) \\
13(24.5)\end{array}$ & $\begin{array}{l}0.019 \\
0.002\end{array}$ \\
\hline IPSS total & $17.9 \pm 8.9$ & $17.5 \pm 9.4$ & $18.3 \pm 8.8$ & 0.722 \\
\hline IPSS storage subscore & $7.3 \pm 3.9$ & $7.1 \pm 4.3$ & $7.4 \pm 3.7$ & 0.750 \\
\hline IPSS voiding subscore & $10.7 \pm 5.6$ & $10.3 \pm 5.6$ & $10.9 \pm 5.7$ & 0.662 \\
\hline IPSS QoL score & $3.9 \pm 1.1$ & $3.7 \pm 1.2$ & $4.0 \pm 0.9$ & 0.329 \\
\hline \multicolumn{5}{|l|}{ Urodynamic parameters } \\
\hline $\operatorname{Qmax}(\mathrm{mL} / \mathrm{sec})$ & $8.4 \pm 4.0$ & $7.7 \pm 3.7$ & $8.9 \pm 4.2$ & 0.147 \\
\hline $\operatorname{PVR}(\mathrm{mL})$ & $57.1 \pm 74.9$ & $58.9 \pm 62.6$ & $55.9 \pm 82.2$ & 0.857 \\
\hline Maximum bladder capacity $(\mathrm{mL})$ & $364.6 \pm 121.5$ & $361.1 \pm 139.9$ & $366.7 \pm 109.8$ & 0.838 \\
\hline $\operatorname{MUCP}\left(\mathrm{cm} \mathrm{H}_{2} \mathrm{O}\right)$ & $81.9 \pm 35.8$ & $77.6 \pm 36.9$ & $84.8 \pm 35.5$ & 0.383 \\
\hline $\operatorname{PdetQmax}\left(\mathrm{cm} \mathrm{H}_{2} \mathrm{O}\right)$ & $56.5 \pm 30.7$ & $60.2 \pm 32.4$ & $54.1 \pm 29.6$ & 0.379 \\
\hline BOOI & $41.8 \pm 31.4$ & $46.7 \pm 35.4$ & $38.6 \pm 28.4$ & 0.248 \\
\hline \multicolumn{5}{|l|}{ BOO grade } \\
\hline $3-6$ & $47(54.7)$ & $16(48.5)$ & $31(58.5)$ & 0.365 \\
\hline $0-2$ & $39(45.3)$ & $17(51.5)$ & $22(41.5)$ & \\
\hline BCI & $96.2 \pm 39.5$ & $100.3 \pm 34.3$ & $93.6 \pm 42.6$ & 0.449 \\
\hline
\end{tabular}

Values are presented as mean \pm standard deviation or number (\%).

SUI, stress urinary incontinence; AUR, acuter urinary retention; PSA, prostate specific antigen; IPSS, international prostate symptom score; QoL, quality of life; Qmax, maximum flow rate; PVR, postvoid residual; MUCP, maximum urethral closure pressure; PdetQmax, detrusor pressure on maximum flow; BOO, bladder outlet obstruction; BOOI, bladder obstruction index; BCI, bladder contractility index.

Table 2. Comparison of perioperative results between early SUI recovery group and persistent SUI group

\begin{tabular}{lcccc}
\hline Variable & Total $(\mathrm{n}=86)$ & Early SUI recovery $(\mathrm{n}=33)$ & Persistent SUI $(\mathrm{n}=53)$ & P-value \\
\hline Enucleation time (min) & $51.8 \pm 23.9$ & $57.6 \pm 26.8$ & $48.3 \pm 21.6$ & 0.159 \\
Morcellation time (min) & $8.0 \pm 5.4$ & $8.3 \pm 6.8$ & $7.8 \pm 4.4$ & 0.069 \\
Used energy (KJ) & $139.3 \pm 62.8$ & $157.9 \pm 69.9$ & $128.5 \pm 56.3$ & 0.819 \\
Enucleation weight (g) & $22.2 \pm 16.1$ & $22.0 \pm 14.2$ & $22.4 \pm 17.2$ & 0.307 \\
Enucleation efficiency (g/min) & $0.45 \pm 0.29$ & $0.42 \pm 0.29$ & $0.47 \pm 0.30$ & 0.437 \\
Morcellation efficiency (g/min) & $3.02 \pm 1.83$ & $3.18 \pm 1.65$ & $2.92 \pm 1.94$ & 0.530 \\
Enucleation ratio & $0.78 \pm 0.42$ & $0.63 \pm 0.29$ & $0.87 \pm 0.46$ & 0.010 \\
\hline
\end{tabular}

Values are presented as mean \pm standard deviation.

SUI, stress urinary incontinence.

$\mathrm{TZ}$ volume $<30 \mathrm{~mL}$ in the persistent SUI group than in the early SUI recovery group. Perioperative factors are presented in Table 2. The enucleation ratio in the early SUI recovery group was significantly lower than in the persistent $\mathrm{SUI}$ group $(\mathrm{P}=$ 0.010). Multivariate analysis showed that the $\mathrm{TZ}$ volume and the enucleation ratio were significantly associated with early recovery of SUI (Table 3). Surgical outcomes evaluated 1 month after HoLEP showed similar results between the 2 groups, except for the IPSS total score, storage subscore, and quality of life score (Table 4). 
Table 3. Multivariate analyses of factors affecting early recovery of stress urinary incontinence following holmium laser enucleation of prostate

\begin{tabular}{lcc}
\hline Variable & Odds ratio $(95 \% \mathrm{CI})$ & P-value \\
\hline TZ prostate volume & $5.354(1.911-14.999)$ & 0.001 \\
Enucleation ratio & $8.253(1.786-38.126)$ & 0.007 \\
\hline
\end{tabular}

CI, confidence interval; TZ, transition zone.

\section{DISCUSSION}

SUI following HoLEP is an important consideration that may negatively affect patients' daily activities. The timing of SUI recovery following HoLEP is important for improving quality of life and treatment satisfaction. In this study, we identified factors associated with early recovery of SUI, defined as recovery occurring within 1 month after HoLEP.

SUI following HoLEP occurred in approximately $22 \%$ of all patients. This is a higher incidence rate than has been reported in previous studies, possibly because continence was defined as complete dryness in any situation that increased abdominal pressure. Although we did not conduct a pad test for all patients in this study, most patients had mild SUI and used $\leq 2$ pads per day. Nevertheless, the timing of SUI recovery varied and patients with SUI felt uncomfortable. In this study, roughly $40 \%$ of patients with SUI following HoLEP recovered from SUI within 1 month, approximately $40 \%$ recovered between 1 and 3 months, and SUI persisted for longer than 3 months in about $20 \%$.

Continence in males is maintained by components of the urethral sphincter complex. The internal lissosphincter of smooth muscle and the external rhabdosphincter of skeletal muscle are responsible for continence at rest and during stress conditions, respectively [12]. Possible mechanisms of de novo SUI after prostatectomy for BPE have been suggested. The external urethral sphincter can be damaged by resectoscope sheath manipulation across the external urethral sphincter or by unintended resection during the operation. The internal urethral sphincter can also be damaged during prostatectomy. In addition, an enlarged prostate may prevent urine leakage and weaken the external urethral sphincter by compressing the urethra. A large prostatic fossa, created by removing more prostatic tissue, might hold more urine. Thus, more complete removal of prostatic adenomas may be related to SUI [13]. The enucleation ratio was significantly higher in the persistent SUI group than in the early SUI recovery group in this study. A high enucle-
Table 4. Comparison of postoperative voiding parameters at 1 month

\begin{tabular}{lccc}
\hline Variable & $\begin{array}{c}\text { Early SUI recovery } \\
(\mathrm{n}=33)\end{array}$ & $\begin{array}{c}\text { Persistent SUI } \\
(\mathrm{n}=51)\end{array}$ & P-value \\
\hline Qmax $(\mathrm{mL} / \mathrm{sec})$ & $14.1 \pm 6.0$ & $13.7 \pm 7.4$ & 0.816 \\
PVR $(\mathrm{mL})$ & $39.7 \pm 86.1$ & $21.0 \pm 19.9$ & 0.252 \\
IPSS & & & \\
Total & $11.5 \pm 7.8$ & $16.9 \pm 9.5$ & 0.012 \\
Storage subscore & $6.5 \pm 4.0$ & $9.3 \pm 4.1$ & 0.008 \\
Voiding subscore & $4.9 \pm 4.5$ & $7.6 \pm 6.7$ & 0.051 \\
QoL score & $2.6 \pm 1.6$ & $4.1 \pm 1.4$ & $<0.001$ \\
\hline
\end{tabular}

Values are presented as mean \pm standard deviation.

SUI, stress urinary incontinence; Qmax, maximum flow rate; PVR, postvoid residual; IPSS, international prostate symptom score; QoL, quality of life.

ation ratio indicates that more of the $\mathrm{TZ}$ was removed by HoLEP, which might be consistent with the proposal that more thorough removal of prostatic adenomas can be a mechanism of SUI. More extensive removal of prostatic adenomas may affect the recovery time of SUI. SUI caused by other mechanisms might recover earlier. HoLEP might result in more radical removal of adenomas than TURP, as indicated by its high complete enucleation rate [14]. This may reflect differences in the presence and recovery of postoperative SUI between HoLEP and TURP. It is necessary to explore why the enucleation ratio differed between the 2 groups in our study. The difference was not due to surgical technique, because there were no significant differences in perioperative factors, such as operation time, laser energy used, enucleation efficiency, and morcellation efficiency. Differences in the enucleation ratio may be related to $\mathrm{TZ}$ volume. The TZ volume of the prostate in the early SUI recovery group was higher than that in the persistent SUI group in this study. Although the accuracy of transrectal ultrasonography for measuring $\mathrm{TZ}$ volume compared to the use of enucleated adenoma weight is controversial, TZ volume corresponds to the extent of adenomas removed by surgical treatment for BPE. TZ volume may be correlated with the symptoms of BPE and response to treatment $[15,16]$. Various lobar classifications of BPE based on the TZ in magnetic resonance imaging have been proposed [17]. Although we did not investigate the morphological patterns of BPE in this study, various patterns of BPE can affect adenoma enucleation and cause differences in $\mathrm{TZ}$ volume from case to case. A TZ volume $<30 \mathrm{~mL}$ was associated with persistent SUI following HoLEP in this study. Patients with a low TZ volume may therefore be candidates for 
other surgical techniques, such as bipolar TURP or even transurethral incision of the prostate, to promote early recovery of SUI after surgery.

Our results do not imply that the enucleation ratio should be lowered to increase the likelihood of early recovery from SUI following HoLEP. No significant difference was found in postoperative voiding parameters such as Qmax, PVR, and the IPSS voiding subscore between the 2 groups. A meta-analysis showed no significant difference between TURP and HoLEP in terms of functional outcomes [18]. However, those results were not based on long-term follow-up data, and it is important to consider whether complete adenoma removal can prevent the recurrence of BPE. It is also difficult to intentionally lower the enucleation ratio while conducting HoLEP. We suggest that patients who are expected to have delayed SUI recovery following HoLEP based on the enucleation ratio should be treated more aggressively and systematically. Most patients with SUI are recommended to undergo conservative treatment such as pelvic floor exercises, as physicians do not start pharmacotherapy early. Overactive bladder or mixed urinary incontinence after prostatic surgery can be managed with medication, but there is no approved pharmacotherapy for SUI after prostate surgery. However, Cornu et al. [19] reported that duloxetine reduced incontinence episodes and improved the quality of life of patients with mild to moderate SUI after radical prostatectomy. Although more evidence from additional studies is needed, early management is likely to be helpful for SUI patients at risk for delayed SUI recovery.

Our study has some limitations. First, it enrolled a relatively small number of patients and the data analysis was retrospective. Therefore, further evaluation and validation of our study findings are required. Second, HoLEP was not performed by a single surgeon and the incidence of SUI was not compared between the 2 surgeons. However, only cases that were performed after the surgeons completed the learning curve were included and surgical techniques at a single center would not significantly differ. Thus, little difference would be expected in the functional outcomes between the 2 surgeons. Another limitation is that insufficient postoperative objective data support our conclusions; such data would include postoperative ultrasonographic findings of the prostate and urodynamic findings. However, if significant postoperative problems do not occur, physicians generally do not perform such tests.

Only $40 \%$ of patients with SUI following HoLEP recovered within 1 month, and the TZ volume and enucleation ratio were significantly associated with early SUI recovery following HoLEP. Adequate patient selection for HoLEP may be helpful for promoting early recovery of SUI after HoLEP. In addition, patients with risk factors for persistent SUI following HoLEP require early and active conservative management to improve their quality of life.

\section{AUTHOR CONTRIBUTION STATEMENT}

- Full access to all the data in the study and takes responsibility for the integrity of the data and the accuracy of the data analysis: JCK

- Study concept and design: JCK

- Acquisition of data: JSK, JBC

- Analysis and interpretation of data: KJC

- Drafting of the manuscript: KJC

- Critical revision of the manuscript for important intellectual content: JCK

- Statistical analysis: $J B C$

- Administrative, technical, or material support: JSK

-Study supervision: JCK

\section{REFERENCES}

1. Arai Y, Kaiho Y, Takei M, Nonomura K, Baba S, Habuchi T, et al. Burden of male stress urinary incontinence: a survey among urologists in Japan. Int J Urol 2009;16:915-7.

2. Serretta V, Morgia G, Fondacaro L, Curto G, Lo bianco A, Pirritano

$\mathrm{D}$, et al. Open prostatectomy for benign prostatic enlargement in southern Europe in the late 1990s: a contemporary series of 1800 interventions. Urology 2002;60:623-7.

3. Tubaro A, Carter S, Hind A, Vicentini C, Miano L. A prospective study of the safety and efficacy of suprapubic transvesical prostatectomy in patients with benign prostatic hyperplasia. J Urol 2001; 166:172-6.

4. Montorsi F, Naspro R, Salonia A, Suardi N, Briganti A, Zanoni M, et al. Holmium laser enucleation versus transurethral resection of the prostate: results from a 2-center, prospective, randomized trial in patients with obstructive benign prostatic hyperplasia. J Urol 2004;172(5 Pt 1):1926-9.

5. Elmansy HM, Kotb A, Elhilali MM. Is there a way to predict stress urinary incontinence after holmium laser enucleation of the prostate? J Urol 2011;186:1977-81.

6. Vavassori I, Valenti S, Naspro R, Vismara A, Dell'Acqua V, Manzetti A, et al. Three-year outcome following holmium laser enucleation 
of the prostate combined with mechanical morcellation in 330 consecutive patients. Eur Urol 2008;53:599-604.

7. Krambeck AE, Handa SE, Lingeman JE. Experience with more than 1,000 holmium laser prostate enucleations for benign prostatic hyperplasia. J Urol 2010;183:1105-9.

8. Kobayashi S, Yano M, Nakayama T, Kitahara S. Predictive risk factors of postoperative urinary incontinence following holmium laser enucleation of the prostate during the initial learning period. Int Braz J Urol 2016;42:740-6.

9. Lerner LB, Tyson MD, Mendoza PJ. Stress incontinence during the learning curve of holmium laser enucleation of the prostate. J Endourol 2010;24:1655-8.

10. Shigemura K, Tanaka K, Yamamichi F, Chiba K, Fujisawa M. Comparison of predictive factors for postoperative incontinence of $\mathrm{Hol}-$ mium laser enucleation of the prostate by the surgeons' experience during learning curve. Int Neurourol J 2016;20:59-68.

11. Gilling P. Holmium laser enucleation of the prostate (HoLEP). BJU Int 2008;101:131-42.

12. Koraitim MM. The male urethral sphincter complex revisited: an anatomical concept and its physiological correlate. J Urol 2008;179:16839.

13. Cho MC, Park JH, Jeong MS, Yi JS, Ku JH, Oh SJ, et al. Predictor of de novo urinary incontinence following holmium laser enucleation of the prostate. Neurourol Urodyn 2011;30:1343-9.
14. Cho SY, Park J, Yoo S, Cho MC, Jeong H, Son H. One-year surgical outcomes of complete or incomplete enucleation of prostate by monopolar electrocoagulation, photoselective vapoenucleation of 120 W GreenLight Laser, and Holmium Laser. Urology 2017;108:142-8.

15. Guneyli S, Ward E, Peng Y, Nehal Yousuf A, Trilisky I, Westin C, et al. MRI evaluation of benign prostatic hyperplasia: Correlation with international prostate symptom score. J Magn Reson Imaging 2017;45:917-25.

16. Tewari A, Shinohara K, Narayan P. Transition zone volume and transition zone ratio: predictor of uroflow response to finasteride therapy in benign prostatic hyperplasia patients. Urology 1995;45:258-64.

17. Wasserman NF, Spilseth B, Golzarian J, Metzger GJ. Use of MRI for lobar classification of benign prostatic hyperplasia: potential phenotypic biomarkers for research on treatment strategies. AJR Am J Roentgenol 2015;205:564-71.

18. Qian X, Liu H, Xu D, Xu L, Huang F, He W, et al. Functional outcomes and complications following B-TURP versus HoLEP for the treatment of benign prostatic hyperplasia: a review of the literature and Meta-analysis. Aging Male 2017;20:184-91.

19. Cornu JN, Merlet B, Ciofu C, Mouly S, Peyrat L, Sèbe P, et al. Duloxetine for mild to moderate postprostatectomy incontinence: preliminary results of a randomised, placebo-controlled trial. Eur Urol 2011;59:148-54. 